\title{
Coupled cluster analysis of the U(1) lattice gauge model using a correlated "mean-field" reference state
}

\author{
S. J. Baker, R. F. Bishop, ${ }^{*}$ and N. J. Davidson ${ }^{\dagger}$ \\ Department of Physics, University of Manchester Institute of Science and Technology (UMIST), P.O. Box 88, \\ Manchester M60 1QD, United Kingdom
}

(Received 16 October 1995)

\begin{abstract}
We apply the coupled cluster method (CCM) to the U(1) lattice gauge model in $2+1$ dimensions, basing our analysis on a reference state which already contains a certain class of single-plaquette correlations, the socalled "mean-field" reference state. We consider the simplest possible approximation scheme for the coupled cluster calculation, in which only single-plaquette correlations are included. The use of the "mean-field" reference state allows us to reproduce, within this level of approximation, the correct leading-order behavior of the ground-state energy of the equivalent Mathieu problem in both the strong- and weak-coupling limits. In addition, we have calculated the mean plaquette and the odd- and even-parity mass gaps.
\end{abstract}

PACS number(s): 11.15.Ha, 03.65.Ca, 11.15.Tk

\section{INTRODUCTION}

Although most of the effort in the field of lattice gauge theories (LGT) has been put into the Lagrangian formulation, there are several advantages to consider these theories in the Hamiltonian formulation [1]. In particular, the Hamiltonian approach can remove the need to extrapolate the results obtained on finite lattices to the infinite lattice limit. It also leaves the time dimension continuous, so that the extrapolation to the continuum limit need only be performed in the spatial dimensions. A further advantage of the Hamiltonian formalism is that it permits a direct application of the techniques of quantum many-body theory (QMBT) to the study of LGT systems.

QMBT has undergone rapid progress over the last decade, with particular advances in the method of correlated basis functions (CBF's) [2] and the coupled cluster method (CCM) [3]. Both these methods have been applied to a vast array of problems in many-body physics, and, more recently, to lattice gauge theories [4-6], in particular $\mathrm{U}(1)$ and $Z_{2}$. A method related to the CCM has also been applied to $\mathrm{SU}(2)$ [7].

The $\mathrm{U}(1)$ model in $2+1$ dimensions has served as a testing ground for many techniques in LGT, among them the CCM $[5,6]$, the CBF method [4], Páde approximant extensions to strong-coupling series $[8,9]$, variational techniques [10-14], and the so-called $t$ expansion [15]. Although the $\mathrm{U}(1)$ model is comparatively simple, it is strongly nonperturbative and as such provides a real test for the various methods.

The CCM has proved its worth as a method of QMBT in fields as diverse as prototypical field theories, spin-lattice systems of interest in magnetism, and quantum chemistry (for a review, see Ref. [16]). In a previous paper [5], the $\mathrm{CCM}$ has been applied to the U(1) model with very encour-

\footnotetext{
*Electronic address: R.F.Bishop@umist.ac.uk

${ }^{\dagger}$ Present address: Department of Physics, University of Pretoria, Pretoria 0002, South Africa.
}

aging results. However, the approach outlined in this earlier work was better suited to the strong-coupling region, and considerably more numerical effort was required to obtain accurate results in the weak-coupling regime. In the present paper we present an alternative approach to the problem which reproduces the correct behavior in both limits with minimal numerical effort (although without using this knowledge as input), and gives very good results in the intermediate-coupling region as well.

The paper is arranged as follows. In Sec. II, we provide a brief outline of our approach, stressing in particular the areas in which it differs from that of Ref. [5]. In Secs. III and IV, the approximations which we employ are described, while our results for several ground-state properties and the mass gaps are presented and discussed in Sec. V. Section VI contains our conclusions and a discussion of future work.

\section{COUPLED CLUSTER FORMALISM FOR THE U(1) MODEL}

Since the general formalism for applying the CCM to lattice gauge theories has been discussed at length in Ref. [5], we shall keep the present discussion brief and only expand where the present work differs from that of the earlier literature.

For $\mathrm{U}(1)$ theory in $1+1$ dimensions [the one-dimensional (1D) chain] and 2+1 dimensions (the 2D square lattice), it is possible to write the Hamiltonian entirely in terms of the plaquette variables $\left\{B_{p}\right\}$ :

$$
H=\sum_{p}\left[-2 \frac{\partial^{2}}{\partial B_{p}^{2}}+\lambda\left(1-\cos B_{p}\right)\right]+\frac{1}{2} \sum_{p, q} \frac{\partial^{2}}{\partial B_{p} \partial B_{p+q}},
$$

where the sum on $p$ runs over all elementary plaquettes, and the sum on $q$ over all lattice vectors connecting nearestneighbor plaquettes on the lattice. Thus, $q$ takes two values for the $1 \mathrm{D}$ chain, and four for the 2D square lattice. Since this Hamiltonian is invariant under the transformation $\left\{B_{p}\right\} \rightarrow\left\{B_{p}+2 n \pi\right\}$, with $n$ integral, the space of $B_{p}$ is compact with $-\pi \leqslant B_{p} \leqslant \pi$ for all $p$. 
In what follows, we will require the definition of an inner product of two functions of the plaquette variables. For functions $\left\langle\tilde{g}\left(\left\{B_{p}\right\}\right)\right|$ and $\left|f\left(\left\{B_{p}\right\}\right)\right\rangle$, we define the inner product as

$$
\langle\tilde{g} \mid f\rangle=\int_{-\pi}^{\pi}\left[\prod_{p=1}^{N_{p}} \frac{d B_{p}}{2 \pi}\right] \tilde{g} f .
$$

In the limit of a single plaquette, the Schrödinger equation corresponding to the Hamiltonian of Eq. (1) reduces to the one-body Mathieu equation. In previous work [5], the reference state for the CCM analysis was taken as the strongcoupling $(\lambda=0)$ ground state of the Mathieu equation, i.e., as a constant. The correlation operators were then expanded in terms of the excited $\lambda=0$ eigenstates of the Mathieu equation, which are simply the trigonometric functions $\cos n B$ and $\sin n B, n=1,2, \ldots$

The use of the strong-coupling Mathieu ground state as the reference state means that it becomes progressively more difficult to obtain converged results as $\lambda$ increases into the weak-coupling regime. In the present work, we use a different reference state which allows us to obtain much improved convergence throughout the range $0 \leqslant \lambda<\infty$.

If we denote the constant reference state by $|0\rangle$, we define our new reference state as

$$
|\Phi\rangle=e^{T}|0\rangle
$$

The operator $T$ is defined to be $T=t \Sigma_{p} \cos B_{p}$, where $t$ is a parameter to be determined by some external means (e.g., variationally). In the sense that some one-plaquette correlations are included in this state, $|\Phi\rangle$ can be viewed as a "mean-field" (MF) reference state. If we assume that the state $|0\rangle$ is normalized to unity, then the "mean-field" reference state $|\Phi\rangle$ is normalized to $\left[I_{0}(2 t)\right]^{N_{p}}$, where $N_{p}$ is the total number of plaquettes on the lattice and $I_{0}$ is the zerothorder modified Bessel function.

The exact ground-state ket of the Hamiltonian of Eq. (1) is now taken in the CCM form

$$
\left|\Psi_{0}\left(\left\{B_{p}\right\}\right)\right\rangle=e^{S\left(\left\{B_{p}\right\}\right)}|\Phi\rangle,
$$

with

$$
S\left(\left\{B_{p}\right\}\right)=\sum_{k=1}^{N_{p}} S_{k}\left(\left\{B_{p}\right\}\right) .
$$

The $k$-body correlation operators $S_{k}\left(\left\{B_{p}\right\}\right)$ are decomposed as

$$
S_{1}=\sum_{n=1}^{\infty} \sum_{p=1}^{N_{p}} \mathscr{S}_{p}(n)\left(\cos n B_{p}-R_{n}\right)
$$

$$
\begin{aligned}
S_{2}= & \frac{1}{2 !} \sum_{n_{1}, n_{2}=1}^{\infty} \sum_{p_{1}, p_{2}=1}^{N_{p}}{ }^{\prime}\left[\mathscr{S}_{p_{1} p_{2}}^{(1)}\left(n_{1}, n_{2}\right)\left(\cos n_{1} B_{p_{1}}-R_{n_{1}}\right)\right. \\
& \times\left(\cos n_{2} B_{p_{2}}-R_{n_{2}}\right) \\
& \left.+\mathscr{S}_{p_{1} p_{2}}^{(2)}\left(n_{1}, n_{2}\right) \sin n_{1} B_{p_{1}} \sin n_{2} B_{p_{2}}\right]
\end{aligned}
$$

with obvious generalization for $k>2$. In Eq. (7) $R_{n} \equiv I_{n}(2 t) / I_{0}(2 t)$ where $I_{n}$ is the $n$ th-order modified Bessel function. The prime on the plaquette summation in $S_{2}$ indicates the omission of the term $p_{1}=p_{2}$. The construction of $|\Psi\rangle$ in the above way ensures that the ground state has positive parity under the simultaneous reversal of the signs of all the $\left\{B_{p}\right\}$.

The $\left\{S_{k}\right\}$ operators defined above differ from those defined in Ref. [5] for the constant reference state in the replacement $\cos n B_{p} \rightarrow \cos n B_{p}-R_{n}$. The reason for this replacement is the following. In the CCM, the correlation operators $\left\{S_{k}\right\}$ are defined in terms of the creation operators relative to some reference state $|\phi\rangle$. We should thus have $\left\langle\phi\left|S_{k}\right| \phi\right\rangle=0$ for each $k$. If we use $|0\rangle$ as the reference state, this condition is satisfied if we take the creation operators to be simply the trigonometric functions, because of their periodicity. However, the use of $|\Phi\rangle$ as the reference state means that $\left\langle\Phi\left|\cos n B_{p}\right| \Phi\right\rangle=I_{n}(2 t)\left[I_{0}(2 t)\right]^{N_{p}-1}$. The presence of the term $-R_{n}$ in Eq. (7) is to correct for this. Note that no change is necessary for the odd parity operators $\sin n B_{p}$, as $\left\langle\Phi\left|\sin n B_{p}\right| \Phi\right\rangle$ vanishes on account of the odd character of $\sin n B_{p}$ under the substitution $B_{p} \rightarrow-B_{p}$ and the fact that the inner product involves integration over a symmetric interval.

In the CCM the ground-state bra $\left\langle\tilde{\Psi}_{0}\right|$ is not the manifest Hermitian conjugate of the ket, but is parametrized independently in the form [16]

$$
\left\langle\tilde{\Psi}_{0}\right|=\langle\Phi| \tilde{S}\left(\left\{B_{P}\right\}\right) e^{-S\left(\left\{B_{p}\right\}\right)}
$$

where $S$ is as defined above for the ground-state ket. The operator $\tilde{S}$ is given by

$$
\tilde{S}=1+\sum_{k=1}^{N_{p}} \tilde{S}_{k}\left(\left\{B_{p}\right\}\right)
$$

and $\tilde{S}_{k}$ has the same form as $S_{k}$ except that all coefficients $\mathscr{S}_{p_{1} \ldots}\left(n_{1}, \ldots\right)$ are replaced by $\tilde{\mathscr{S}}_{p_{1} \ldots}\left(n_{1}, \ldots\right)$.

The coefficients $\mathscr{S}_{p_{1}} \ldots\left(n_{1}, \ldots\right)$ and $\tilde{\mathscr{S}}_{p_{1} \ldots}\left(n_{1}, \ldots\right)$ are determined from the CCM equations [5] which are obtained from the condition that the ground-state expectation value of the Hamiltonian,

$$
\bar{H}=\frac{\left\langle\tilde{\Psi}_{0}|H| \Psi_{0}\right\rangle}{\left\langle\tilde{\Psi}_{0} \mid \Psi_{0}\right\rangle}=\frac{\left\langle\Phi\left|\tilde{S} e^{-S} H e^{S}\right| \Phi\right\rangle}{\langle\Phi \mid \Phi\rangle},
$$

should be stationary with respect to variations in both the bra- and ket-state coefficients.

The CCM can be extended to the determination of the excited states of a system as well as its ground-state properties. In the conventional CCM, the excited states are constructed by acting on the ground state with an excitation correlation operator $X$, which has a similar partitioning to $S$ for excited states which have the same symmetry as the 
ground state; for excited states with a different symmetry [e.g., parity, as in our U(1) case], the excitation correlation operator for these states must reflect their underlying symmetry. The Schrödinger equation for the excited state $\left|\Psi_{e}\right\rangle$ is then written as

$$
H\left|\Psi_{e}\right\rangle=E_{e}\left|\Psi_{e}\right\rangle
$$

with

$$
\left|\Psi_{e}\right\rangle=X\left|\Psi_{0}\right\rangle=X e^{S}|\Phi\rangle
$$

\section{THE “MEAN-FIELD” APPROXIMATION}

The simplest approximation scheme available is the "mean-field" scheme, in which we set $S=0$ and $\tilde{S}=1$. We thus approximate the exact ground-state ket and bra by

$$
\left|\Psi_{0}\right\rangle=|\Phi\rangle ;\left\langle\tilde{\Psi}_{0}\right|=\langle\Phi|
$$

and the expectation value of the Hamiltonian by

$$
\bar{H}^{\mathrm{MF}}=\frac{\langle\Phi|H| \Phi\rangle}{\langle\Phi \mid \Phi\rangle}=N_{p}\left(\lambda+[t-\lambda] R_{1}\right) .
$$

There is thus only one parameter, namely $t$, to determine, and we do this by requiring that $\bar{H}$ should be stationary with respect ${ }^{1}$ to $t$. This value of $t$, which we denote by $t_{0}$, is given by the solution of the equation

$$
\lambda=t+\frac{R_{1}}{R_{1}^{\prime}}
$$

where the prime denotes differentiation with respect to $t$. In our subsequent calculations, where $S \neq 0$ and $(\tilde{S}-1) \neq 0$, we shall choose $t_{0}$ to satisfy Eq. (15), i.e., to minimize the "mean-field" estimate ${ }^{2}$ of $\bar{H}^{\mathrm{MF}}$ of Eq. (14).

The advantage of using $|\Phi\rangle$ rather than $|0\rangle$ as a reference state becomes clear from an examination of the strongcoupling $(\lambda \rightarrow 0)$ and weak-coupling $(\lambda \rightarrow \infty)$ limits of Eq. (14). If we denote $\bar{H}^{\mathrm{MF}}\left(t=t_{0}\right)$ by $E_{g}^{\mathrm{MF}}$, we find in the former case (to lowest order) that

$$
\frac{E_{g}^{\mathrm{MF}}}{N_{p}} \sim \lambda-\frac{\lambda^{2}}{4}+O\left(\lambda^{4}\right),
$$

which agrees with strong-coupling perturbation theory for the Mathieu problem [and, in fact, with the U(1) result in any dimension] to the order shown. In the case of weak coupling, we find that

$$
\frac{E_{g}^{\mathrm{MF}}}{N_{p}} \sim C_{0} \sqrt{\lambda}-\frac{C_{0}^{2}}{8}+O\left(\lambda^{-1 / 2}\right)
$$

\footnotetext{
${ }^{1}$ At this level of approximation $(S=0, \tilde{S}=1)$, we are essentially repeating the variational calculation of Ref. [10].

${ }^{2}$ Note that the last term of the Hamiltonian of Eq. (1) does not contribute to the calculation of $\bar{H}$ in Eq. (14), which means that we are effectively solving a Mathieu-type problem.
}

with $C_{0}=1$. This agrees with the result of weak-coupling perturbation theory for the Mathieu problem to the order shown. [It should be noted that the U(1) problem has a similar weak-coupling expansion, with $C_{0}=0.9833$ for the $1 \mathrm{D}$ chain [17] and $C_{0}=0.9581$ for the $2 \mathrm{D}$ square lattice [4].]

Since we obtain the correct behavior in these two limits without the addition of any correlations apart from those already included in $T$, we should be able to obtain very good results at low orders of approximation in the CCM calculations throughout the range of $\lambda$. This emphasizes the nonperturbative nature of the CCM, since both the Mathieu and $\mathrm{U}(1)$ problems are well known for lack of convergence in their strong-coupling perturbation expansions.

The fact that this very low-order "mean-field" approximation, which includes only some one-body correlations, leads to results for the ground-state energy in the weakcoupling regime which are less than $2 \%$ in error for 1D and less than $5 \%$ in error for $2 \mathrm{D}$ suggests that the higher-order correlations play a small role in determining the ground-state energy of these $U(1)$ lattice systems.

In previous work [5], the connection between the CCM and strong-coupling perturbation theory for the U(1) model was stressed. The use of a constant reference state in that work made this analysis simple and straightforward. In the present work, the use of the more complex reference state $|\Phi\rangle$ makes this analysis more complicated, even for low orders. This is because of the relationship of Eq. (15), which only allows analytic solution for very low orders. For this reason, it is not readily feasible to replicate the high-order comparisons between the various $\operatorname{SUB} 1(m)$ approximations and $2 m$ th order perturbation theory as was done in Ref. [5].

\section{THE ONE-PLAQUETTE APPROXIMATION SCHEME}

\section{A. Ground state}

The formulation described above is in principle exact if all the correlation operators $\left\{S_{k}, \tilde{S}_{k} ; k=1,2, \ldots, N_{p}\right\}$ are included in the calculation. However, since we are ultimately interested in the situation where $N_{p} \rightarrow \infty$, this is obviously not a feasible proposition and some form of approximation becomes necessary.

One of the most popular schemes for approximating the full CCM is the so-called SUB $n$ scheme, in which only those correlation operators $\left\{S_{k}, \tilde{S}_{k}\right\}$ with $k \leqslant n$ are retained, and the remainder set to zero. In our subsequent calculations here, we will work in the SUB1 approximation, i.e., $S \equiv S_{1}, \tilde{S} \equiv 1+\tilde{S}_{1}$. While this scheme constitutes a very major approximation, it provides a useful and simple testing ground for properties such as the convergence of the method as a function of the coupling and, by comparison with results from other methods in which the identification of different orders of correlation is not so straightforward, of the importance of higher-order correlations.

Because of the linear dependence of $\bar{H}$ on $\tilde{S}$ in Eq. (10), the CCM equations for the ground-state energy $E_{g}$ and the ket-state coefficients are independent of the bra-state coefficients. Within the SUB1 approximation, $E_{g}$ is given by

$$
E_{g}=\frac{\left\langle\Phi\left|e^{-S_{1}} H e^{S_{1}}\right| \Phi\right\rangle}{\langle\Phi \mid \Phi\rangle}
$$


and the equations for the ket coefficients by

$$
\begin{array}{r}
\left\langle\Phi\left|\cos m B_{p} e^{-S_{1}} H e^{S_{1}}\right| \Phi\right\rangle=E_{g} I_{m}(2 t)\left[I_{0}(2 t)\right]^{N_{p}-1}, \\
m=1,2, \ldots .
\end{array}
$$

We now make the substitution $n \mathscr{S}_{p}(n) \rightarrow a_{n}$, where the $\left\{a_{n}\right\}$ are independent of the plaquette index $p$ because of the translational invariance of the (infinite) lattice. If we extend the definition of the $\left\{a_{n}\right\}$ to include negative values of $n$ with the conventions $a_{-n}=-a_{n}$ and $a_{0}=0$, we can express the ground-state energy per plaquette at the SUB1 level by

$\frac{E_{g}}{N_{p}}=\lambda+(t-\lambda) R_{1}+\frac{1}{2} \sum_{n_{1}=-\infty}^{\infty} \sum_{n_{2}=-\infty}^{\infty} a_{n_{1}} a_{n_{2}} R_{n_{1}+n_{2}}$.

The equations for the ket coefficients are

$$
\begin{aligned}
& \frac{1}{2}(t-\lambda)\left(R_{m+1}+R_{m-1}-2 R_{1} R_{m}\right)+\frac{m^{2}}{2} R_{m} \\
& \quad-m \sum_{n=-\infty}^{\infty} a_{n} R_{n+m}+\frac{1}{2} \sum_{n_{1}=-\infty}^{\infty} \sum_{n_{2}=-\infty}^{\infty} a_{n_{1}} a_{n_{2}} \\
& \quad \times\left(R_{n_{1}+n_{2}+m}-R_{n_{1}+n_{2}} R_{m}\right)=0, \quad m=1,2, \ldots
\end{aligned}
$$

The SUB1 scheme as it stands, however, is still insufficient to allow for exact calculations to be performed because of the infinite sums over the mode indices $n$ which appear in the definitions of $S_{1}$ and $\tilde{S}_{1}$. We therefore introduce an additional subtruncation scheme, the $\operatorname{SUB} 1(m)$ scheme, in which we set the coefficients $a_{n}$ and $\tilde{a}_{n}$ with $n>m$ to zero.

\section{B. Excited states}

We now turn to the parametrization of the excited states within the SUB1 approximation. Since there are two possible branches of excitations for the U(1) model, which are distinguished by their parity under the transformation $\left\{B_{p}\right\} \rightarrow\left\{-B_{p}\right\}$, we require two excitation correlation operators. We will denote the even-parity operator by $X^{e}$ and its odd-parity counterpart by $X^{o}$. Within our SUB1 level of approximation, these operators are given by their one-body partitions

$$
\begin{gathered}
X_{1}^{e}=\sum_{n=1}^{\infty} \sum_{p=1}^{N_{p}} \mathscr{X}_{p}^{e}(n)\left(\cos n B_{p}-R_{n}\right), \\
X_{1}^{o}=\sum_{n=1}^{\infty} \sum_{p=1}^{N_{p}} \mathscr{O}_{p}^{o}(n) \sin n B_{p} .
\end{gathered}
$$

The equations for the single-plaquette excitation coefficients are then given by

$$
\begin{gathered}
\left\langle\Phi\left|\sin m B_{p} e^{-S}\left[H, X^{o}\right] e^{S}\right| \Phi\right\rangle=\epsilon^{o}\left\langle\Phi\left|\sin m B_{p} X^{o}\right| \Phi\right\rangle \\
\left\langle\Phi\left|\left(\cos m B_{p}-R_{m}\right) e^{-S}\left[H, X^{e}\right] e^{S}\right| \Phi\right\rangle \\
=\epsilon^{e}\left\langle\Phi\left|\left(\cos m B_{p}-R_{m}\right) X^{e}\right| \Phi\right\rangle
\end{gathered}
$$

where $\epsilon^{o} \equiv E_{e}^{o}-E_{g}, \epsilon^{e} \equiv E_{e}^{e}-E_{g}$, and $E_{e}^{o}$ and $E_{e}^{e}$ are the energies of the odd- and even-parity excited states.

It is at this point that a major difference between the results of our present work and those of the previous analysis based on the constant reference state appears. If a constant model state is used, all coefficients at the SUB1 level of approximation (i.e., for ground-state bra and ket states, and for even- and odd-parity excited states) are independent of the coordination number of the lattice $z$ (i.e., the number of nearest-neighbor plaquettes for a given plaquette), since the last term in the Hamiltonian (the term $\partial^{2} / \partial B_{p} \partial B_{p+q}$ ) never contributes, and the analysis is essentially a way of solving the Mathieu equation. In our approach with a "mean-field" model state, we find that the odd-parity excitations do depend on $z$, even though the ground state and even-parity excitations are independent of $z$. The set of equations which we have to solve for the coefficients of the even-parity excited states is given by

$$
\begin{aligned}
& -m \sum_{n=-\infty}^{\infty} n x_{n}^{e} R_{m+n} \\
& \quad+\sum_{n_{1}=-\infty}^{\infty} \sum_{n_{2}=-\infty}^{\infty} n_{1} x_{n_{1}}^{e} a_{n_{2}}\left(R_{n_{1}+n_{2}+m}-R_{n_{1}+n_{2}} R_{m}\right) \\
& \quad=\frac{1}{2} \epsilon^{e} \sum_{n=-\infty}^{\infty} x_{n}^{e}\left(R_{m+n}-R_{m} R_{n}\right), \quad m=1,2, \ldots
\end{aligned}
$$

while those for the odd-parity excitations are given by

$$
\begin{aligned}
& m \sum_{n=-\infty}^{\infty} n x_{n}^{o}\left(R_{m+n}-\frac{z}{4} R_{n} R_{m}\right) \\
& \quad+\sum_{n_{1}=-\infty}^{\infty} \sum_{n_{2}=-\infty}^{\infty} n_{1} x_{n_{1}}^{o} a_{n_{2}}\left(\frac{z}{4} R_{n_{1}} R_{m+n_{2}}-R_{n_{1}+n_{2}+m}\right) \\
& =-\frac{1}{2} \epsilon^{o} \sum_{n=-\infty}^{\infty} x_{n}^{o} R_{m+n}, \quad m=1,2, \ldots
\end{aligned}
$$

In the above equations, $x_{n}^{e} \equiv \mathscr{C}_{p}^{e}(n)$ and $x_{n}^{o} \equiv \mathscr{C}_{p}^{o}(n)$; both are independent of the plaquette index because of translational invariance. We have also extended these definitions to include negative values of $n$ using the relations $x_{-n}^{e}=x_{n}^{e}$, $x_{-n}^{o}=-x_{n}^{o}$, and $x_{0}^{o}=x_{0}^{e}=0$. In the derivation of the above equations we have made use of the recurrence relations of the modified Bessel functions [18].

As in the case of the ground state, a truncation of the mode indices is necessary to make the calculation tractable. We can again employ the $\operatorname{SUB} 1(m)$ scheme, in which we retain only those coefficients $a_{n}$ and $x_{n}$ with $n \leqslant m$ in Eqs. (26) and (27). The determinations of $\epsilon^{e}$ and $\epsilon^{o}$ then reduce to the solutions of generalized eigenvalue equations.

The excitation energies which are of particular interest are the lowest excitations in each parity sector, the so-called mass gaps. The lowest excitation in the odd-parity sector (sometimes referred to as the photon-sector mass gap) is related to the glueball mass $M_{A}$ (see below for the precise relationship), where the subscript indicates antisymmetric. 
TABLE I. Ground-state energy per plaquette for various values of $\lambda$ for U(1) lattice gauge theory in $2+1$ dimensions within the $\operatorname{SUB} 1(m)$-approximation scheme for $m=1,2,3,5,10,20$. Within the SUB1 approximation the ground state of the U(1) model is equivalent to the ground state of the Mathieu problem; the highest-order results are exact to the accuracy shown for the Mathieu problem.

\begin{tabular}{lccccccc}
\hline \hline$\lambda$ & 0.5 & 1 & 5 & 10 & 50 & 100 & 200 \\
\hline SUB1(1) & 0.439323 & 0.774584 & 2.130507 & 3.060732 & 6.952695 & 9.879051 & 14.019766 \\
SUB1(2) & 0.439118 & 0.772465 & 2.098194 & 3.026606 & 6.942991 & 9.873201 & 14.015951 \\
SUB1(3) & 0.439117 & 0.772429 & 2.098265 & 3.028447 & 6.943527 & 9.873350 & 14.015996 \\
SUB1(5) & 0.439117 & 0.772431 & 2.099943 & 3.031441 & 6.943734 & 9.873376 & 14.016000 \\
SUB1(10) & 0.439117 & 0.772431 & 2.099977 & 3.031510 & 6.943730 & 9.873375 & 14.016000 \\
SUB1(20) & 0.439117 & 0.772431 & 2.099977 & 3.031510 & 6.943731 & 9.873375 & 14.016000 \\
\hline \hline
\end{tabular}

The even-parity mass gap is related to the physical vacuumsector mass gap $M_{S}(S$ for symmetric).

\section{RESULTS}

In this section we present the numerical results of our CCM SUB1 calculations for the ground-state energy and the mass gaps. In addition to these spectral observables, we have also calculated other quantities which are of interest in the study of lattice gauge theories, namely, the specific heat, Wilson loops, and the mean plaquette.

The specific heat is of interest as it gives some indication of the coupling at which the ground-state energy changes from the power-law behavior at strong coupling to the nonanalytic square-root behavior for weak coupling. Wilson loops and the mean plaquette can be used as order parameters to investigate the phase structure of lattice models [19].

\section{A. Ground-state energy}

The fact that the ground-state energy of the U(1) model under the SUB1 approximation is independent of the lattice coordination number, together with the observation that the last term of the Hamiltonian of Eq. (1) does not contribute to either $E_{g}$ or the ket coefficient equations at this level of approximation, means that at the SUB1 level we are solving the Mathieu problem. The ground-state energy per plaquette, $E_{g} / N_{p}$, is therefore identical to the lowest eigenvalue of the Mathieu equation for a given value of the coupling.

We show our numerical results for $E_{g} / N_{p}$ for the U(1) model under the CCM SUB1 approximation in Table I over a wide range of $\lambda$. It is clear from the results that we obtain good convergence over the entire range of couplings, but particularly in the strong- and weak-coupling limits. The convergence at $\lambda=200$ is very impressive, and stresses the ability of the CCM to perform remarkably well far outside the perturbative region.

It is simple to show that the results obtained for the "mean-field" approximation above, where we showed that the CCM energy in both limits reproduced the relevant loworder perturbation theory expansions, remain true at the SUB1 level, i.e., the additional correlations introduced by $S_{1}$ do not disturb the extreme strong- or weak-coupling behavior.

\section{B. Specific heat}

The ground-state energy in LGT is the analogue of the free energy of statistical mechanics, with a similar analogy between the coupling and the temperature. It is thus customary in LGT to refer to the second derivative of the groundstate energy with respect to the coupling as the specific heat, which we will denote by $\chi$ rather than the more usual $C$ to avoid confusion with contours on the lattice which are introduced below in connection with Wilson loops.

We define $\chi$ as

$$
\chi=-\frac{\partial^{2}}{\partial \beta^{2}}\left(\frac{E_{g}}{\beta N_{p}}\right),
$$

where $\beta=\lambda^{1 / 2}$. We have chosen this definition [15] so as to obtain a peak in $\chi$ as the ground-state energy changes character from strong to weak coupling, rather than the shoulder which would occur if we were to define it in terms of the second derivative with respect to $\lambda[9,20]$.

We show $\chi$ as a function of $\sqrt{\lambda}$ for the SUB1(8) approximation in Fig. 1. There is no discontinuity in $\chi$, which strongly suggests the lack of any phase transition. The peak in $\chi$ is the result of the change from strong to weak coupling, which, according to our results, occurs in the region of $\lambda \approx 0.6$. This is in good agreement with previous results $[10,15]$.

\section{Mass gaps}

The formalism for describing excited states within the $\mathrm{CCM}$ has been described above. In this section, we present the results of our SUB1 calculations for the mass gaps for the $\mathrm{U}(1)$ model in $2+1$ dimensions.

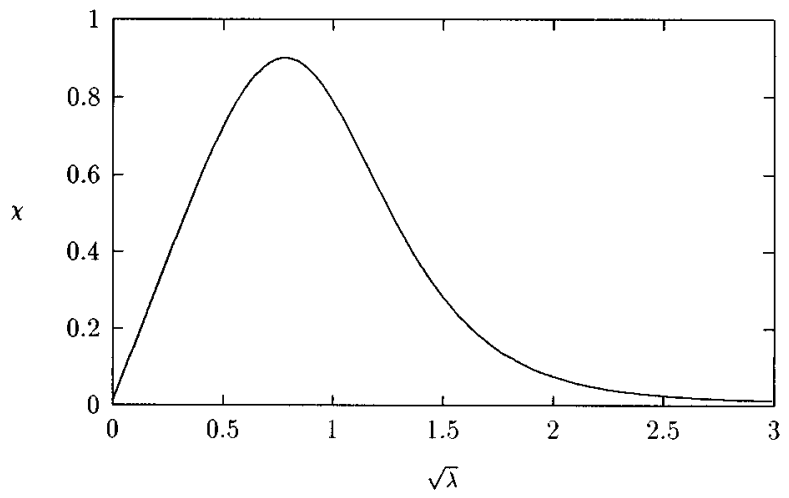

FIG. 1. The specific heat $\chi$ as a function of $\sqrt{\lambda}$ for the SUB1(8) approximation. The peak at $\sqrt{\lambda} \approx 0.78$ is indicative of the change from strong- to weak-coupling behavior. 
TABLE II. Even-parity mass gap for various values of $\lambda$ for $U(1)$ lattice gauge theory in $2+1$ dimensions within the $\operatorname{SUB} 1(m)$-approximation scheme for $m=1,2,3,5,10,20$. Within the SUB1 approximation this mass gap of the U(1) model is equivalent to the first even-parity excitation of the Mathieu problem.

\begin{tabular}{lccccccc}
\hline \hline$\lambda$ & 0.5 & 1 & 5 & 10 & 50 & 100 & 200 \\
\hline SUB1(1) & 2.119021 & 2.422291 & 5.812630 & 9.326872 & 25.156609 & 36.921923 \\
SUB1(2) & 2.110648 & 2.404033 & 6.080411 & 10.163532 & 26.713305 & 38.446453 & 53.517423 \\
SUB1(3) & 2.111321 & 2.412691 & 6.580474 & 11.367925 & 26.753995 & 38.450347 & 55.030397 \\
SUB1(5) & 2.111333 & 2.413220 & 6.634255 & 10.873960 & 26.691813 & 38.440945 & 55.028324 \\
SUB1(10) & 2.111333 & 2.413220 & 6.624583 & 10.827390 & 26.697462 & 38.441374 & 55.028340 \\
SUB1(20) & 2.111333 & 2.413220 & 6.624578 & 10.827175 & 26.697389 & 38.441371 & 55.028340 \\
\hline \hline
\end{tabular}

As was the case for the ground-state energy, the evenparity excitations at the SUB1 level are independent of $z$, so that the mass gap is simply the second-lowest positive parity eigenvalue of the Mathieu equation. For the odd-parity excitations, on the other hand, $z$ does enter the equations [see Eq. (27)]. To reproduce the Mathieu results, we must take $z=0$. The odd-parity mass gap is then the lowest odd-parity eigenvalue of the Mathieu equation. The results of these two calculations are shown in Tables II and III. Although the convergence is not as good as in the case of $E_{g} / N_{p}$, it is still impressive.

The fact that Eq. (27) contains a $z$ dependence means that we expect different behaviors for the two cases $z=0$ (Mathieu) and $z=4$ [U(1) in 2+1 dimensions]. It is well known that $\epsilon^{o}$ for $\mathrm{U}(1)$ in $(2+1) \mathrm{D}$ falls off rapidly with increasing coupling, while for the Mathieu problem it increases, as we have seen in Table III. We have therefore carried out SUB1 calculations with $z=4$.

The physical glueball mass $M$ of the $\mathrm{U}(1)$ system in (2 $+1) \mathrm{D}$ is related to the mass gap $\epsilon$ of the Hamiltonian of Eq. (1) by

$$
M a=\frac{\epsilon}{\sqrt{\lambda}},
$$

where $a$ is the lattice spacing. It is expected that $M$ should decrease exponentially with increasing $\lambda$, according to the expression

$$
M^{2} a^{2} \simeq \sqrt{\lambda} \exp \left[b_{1}+b_{2} \sqrt{\lambda}\right]
$$

In Fig. 2 we therefore show plots of $\ln \left[\left(\epsilon^{o}\right)^{2} / \lambda^{3 / 2}\right]$ against $\sqrt{\lambda}$ for SUB1(4) and SUB1(10) approximations. The predicted exponential decrease with $\lambda$ in the weak-coupling regime is clearly visible.
We have performed least-squares fits to the data of Fig. 2 over the range $1 \leqslant \sqrt{\lambda} \leqslant 2.5$ to determine the coefficients $b_{1}$ and $b_{2}$ in Eq. (30). Our results, together with those from other calculations, are shown in Table IV. Our results are clearly comparable with those obtained by other methods.

One quantity which we cannot predict at the SUB1 level is the ratio of the mass gaps $M_{S} / M_{A}$, since our results for $\epsilon^{e}$ are essentially only for the Mathieu problem. In the weakcoupling limit, this ratio is expected to be $\approx 2$, corresponding to the lack of a stable glueball in the U(1) theory in the limit $\lambda \rightarrow \infty$. To make a prediction for this ratio, correlations beyond the SUB1 level will have to be included in the calculation of the even-parity mass gap.

\section{Wilson loops and the mean plaquette}

We begin by defining the path operator $P_{C}$ for a contour $C$ as

$$
P_{C}=\prod_{p^{\prime}} e^{i B_{p^{\prime}}}
$$

where the product runs over all $N_{C}$ plaquettes which lie inside $C$. The Wilson loop $W_{C}$ is defined as the expectation value of $P_{C}$ in the ground state, so in our CCM analysis it is given by

$$
W_{C}=\left\langle\prod_{p^{\prime}} e^{i B_{p^{\prime}}}\right\rangle=\frac{\left\langle\Phi\left|\tilde{S} \Pi_{p^{\prime}} \exp \left(i B_{p^{\prime}}\right)\right| \Phi\right\rangle}{\langle\Phi \mid \Phi\rangle}
$$

Note that the mean plaquette $U \equiv\left\langle\cos B_{p}\right\rangle$ is simply the smallest Wilson loop, in which $C$ is a single plaquette. At the SUB1 level, we obtain

TABLE III. First odd-parity excitation for various values of $\lambda$ for the Mathieu problem $(z=0)$ within the SUB1 $(m)$-approximation scheme for $m=1,2,3,5,10,20$.

\begin{tabular}{lccccccc}
\hline \hline$\lambda$ & 0.5 & 1 & 5 & 10 & 50 & 100 & 200 \\
\hline SUB1(1) & 2.116739 & 2.395799 & 4.637478 & 6.380933 & 13.848386 & 19.635153 & 27.875817 \\
SUB1(2) & 2.050949 & 2.190696 & 3.978095 & 5.776481 & 13.608417 & 19.478510 & 27.770839 \\
SUB1(3) & 2.050475 & 2.185975 & 3.927026 & 5.741709 & 13.618967 & 19.486238 & 27.774953 \\
SUB1(5) & 2.050477 & 2.186082 & 3.949638 & 5.778249 & 13.622795 & 19.486618 & 27.774994 \\
SUB1(10) & 2.050477 & 2.186082 & 3.949753 & 5.777410 & 13.622566 & 19.486596 & 27.774994 \\
SUB1(20) & 2.050477 & 2.186082 & 3.949753 & 5.777411 & 13.622568 & 19.486596 & 27.774994 \\
\hline \hline
\end{tabular}


TABLE IV. Parameters for the exponential fit of Eq. (30) for the glueball mass. The two coefficients $b_{1}$ and $b_{2}$ are shown for the CCM SUB1(4) and SUB1(10) approximations as well as for the other calculations indicated. Numbers in parentheses indicate uncertainties in the last digit quoted.

\begin{tabular}{lll}
\hline \hline Source & \multicolumn{1}{c}{$b_{1}$} & \multicolumn{1}{c}{$b_{2}$} \\
\hline SUB1(4) & $6.13(3)$ & $-5.39(2)$ \\
SUB1(10) & $6.14(1)$ & $-5.39(1)$ \\
Suranyi [12] & & -5.7 \\
Hamer and Irving [8] & $6.15(43)$ & $-5.3(5)$ \\
Hamer, Oitmaa, and Zheng [9] & $6.27(6)$ & $-5.42(6)$ \\
Heys and Stump [14] & $6.21(6)$ & $-4.97(5)$ \\
Lana [21] & $4.98(10)$ & $-4.1(2)$ \\
Dabringhaus, Ristig, and Clark [4] & $6.26(2)$ & $-4.80(6)$ \\
Morningstar [15] & $5.94(5)$ & $-5.23(4)$ \\
\hline \hline
\end{tabular}

$$
W_{C}=\left[R_{1}\right]^{N_{C}-1}\left[R_{1}+N_{C} \sum_{n=1}^{\infty} \tilde{a}_{n}\left(\frac{R_{n+1}+R_{n-1}}{2}-R_{n} R_{1}\right)\right] .
$$

The Wilson loop serves as a gauge-invariant order parameter to distinguish between a confined phase, where, in the limit of a large loop, it decays according to an area law ( $W_{C} \sim \exp [-K A]$, with $A$ the area enclosed by the loop), and a deconfined phase, where the decay follows a perimeter law ( $W_{C} \sim \exp [-k p]$, with $p$ the perimeter of the loop). Since the $\mathrm{U}(1)$ model in $2+1$ dimensions is confining at all couplings, we expect an area-law behavior everywhere. This is indeed the case, as can easily be seen from Eq. (33) if we take the limit $N_{C} \rightarrow \infty$. In this situation, the prefactor dominates and we obtain

$$
W_{C} \sim\left[R_{1}\right]^{N_{C}}
$$

with a clear area-law behavior.

It is tempting to identify the coefficient $K$ of the area law as the string tension, as was done in Ref. [10]. However, there are dangers in doing this. Our Wilson loops are spacelike rather than timelike, and $K$ is thus not the coefficient of the linear contribution to the static quark-antiquark potential. We will thus avoid making this identification in this work.
The mean plaquette is the simplest gauge-invariant quantity in a pure gauge theory, and, since it can be obtained from the Hellmann-Feynman theorem, as

$$
U=1-\frac{d\left(E_{g} / N_{p}\right)}{d \lambda},
$$

represents the internal energy of the thermodynamic system corresponding to the lattice gauge model. It provides an order parameter for lattice gauge systems in the sense that it exhibits singularities in the bulk thermodynamics at phase transitions, but has the disadvantage that it is always nonzero and thus cannot be used to distinguish phases in terms of it vanishing in one phase and not in another. However, discontinuities in $U$ as a function of the coupling provide a useful means of spotting phase transitions (see, for example, Ref. [22] for the case of U(1) LGT in $3+1$ dimensions, which has a deconfinement transition).

In our calculation there is no indication of any discontinuity in $U$ and thus no suggestion of a phase transition. This is as would be expected for U(1) theory in $2+1$ dimensions [23]. Numerical results for the mean plaquette, as calculated in various $\operatorname{SUB} 1(\mathrm{~m})$ approximations, are shown in Table V and are in good agreement with the results of other methods.

\section{FUTURE WORK AND CONCLUSIONS}

In the present work we have applied the coupled cluster method to the $\mathrm{U}(1)$ model in $2+1$ dimensions. The analysis is an extension of that presented in Ref. [5], in which the convergence of the method has been greatly improved, particularly in the weak-coupling regime, by the use of an improved reference state which includes some one-body correlations. We have worked only at the simplest level of approximation, namely the SUB1 level, where only intermode couplings are considered in the correlation operator, and no account is taken of inter-plaquette correlations. Under the SUB1 scheme, the CCM analysis of the U(1) model essentially reduces to the solution of the Mathieu problem; a notable exception to this general conclusion is the calculation of the odd-parity mass gap, which, even at the SUB1 level, displays a dependence on the lattice coordination number.

The results which we have obtained, even at this low level

TABLE V. Mean plaquette for various values of $\lambda$ for $U(1)$ lattice gauge theory in $2+1$ dimensions within the $\operatorname{SUB} 1(m)$-approximation scheme for $m=1,5,10$. Also shown are results from unistate and bistate $t$ expansions (denoted by $\mathrm{U} t$ and $\mathrm{B} t$, respectively), a guided random-walk algorithm on an $8 \times 8$ lattice (GRW), a Green's function Monte Carlo method (GFMC), and strong- and weak-coupling perturbation theory (PT). Numbers in parentheses indicate uncertainties in the last digit quoted.

\begin{tabular}{llllll}
\hline \hline$\lambda$ & 0.0625 & 0.25 & \multicolumn{1}{c}{1} & \multicolumn{1}{c}{4} \\
\hline SUB1(1) & 0.03122 & 0.12310 & 0.41153 & 0.73964 & 0.87586 \\
SUB1(5) & 0.03122 & 0.12333 & 0.41800 & 0.74416 & 0.87485 \\
SUB1(10) & 0.03122 & 0.12333 & 0.41800 & 0.74415 & 0.87485 \\
U $t[15]$ & $0.03124(4)$ & $0.1238(2)$ & $0.4333(3)$ & $0.757(4)$ & $0.881(3)$ \\
B $t[15]$ & $0.0313(2)$ & $0.123(2)$ & $0.437(3)$ & $0.760(11)$ & $0.882(13)$ \\
GRW [24] & $0.027(3)$ & $0.121(2)$ & $0.439(10)$ & $0.777(12)$ & \\
GFMC [25] & & & $0.433(1)$ & $0.756(1)$ & \\
PT & 0.03123 & 0.12381 & & 0.761 & 0.880 \\
\hline \hline
\end{tabular}


of approximation, are indicative of the power of the CCM and, in particular, its ability to obtain well-converged results far ouside the perturbative regime. Although it is possible to view the CCM as a particularly ingenious resummation of perturbation theory [5], the method is inherently nonperturbative and, as such, has a great advantage over more ad hoc schemes for forcing or accelerating convergence such as Padé approximants. The ability of the CCM to obtain fully converged results for any coupling for the notoriously nonperturbative Mathieu problem to arbitrary accuracy for comparatively little numerical effort is a strong indication of its usefulness in other nonperturbative problems.

One of the advantages of the CCM is that its formulation in terms of correlation operators means that it is comparatively simple to identify the contribution of particular levels of correlations to observables. However, because of the fact that there is no guarantee that the $\mathrm{SUB} n$ series of approximations is smoothly convergent to the exact result, one has to be cautious in making sweeping statements about the relative importance of a particular level of correlation in a calculation. Nevertheless, our results suggest that the ground state of the U(1) system is comparatively weakly correlated. The fact that the ground-state energy of the Mathieu problem is within $5 \%$ of its counterpart for the $\mathrm{U}(1)$ problem in (2 $+1) \mathrm{D}$ in the extreme weak-coupling limit and essentially exact in the extreme strong-coupling limit is already indicative of this. Our SUB1 results for the mean plaquette, which are in good agreement with those of other methods, support this conclusion.

The excited states are rather different, since the Mathieu results for these energies have a completely different behavior to those of the U(1) problem. This is particularly clear for the even-parity excitations, and the inclusion of two-body and higher correlations are obviously necessary if we are to obtain acceptable results for the U(1) system. Nevertheless, our reference state in some sense includes longer-range correlations for the odd-parity mass gap, as shown by its coordination-number dependence in the SUB1 approximation. Our results for $z=4$ are entirely comparable with those of other methods.

The obvious path for future work is the inclusion of interplaquette correlations. A good starting point appears to be the inclusion of two-plaquette correlations via the so-called SUB2- $m$ scheme, in which only correlations between two plaquettes which are $m$ th nearest neighbors or closer are included in $S$. This scheme has been applied with some success to the $\mathrm{U}(1)$ model in $(2+1) \mathrm{D}[5,26]$ using the constant

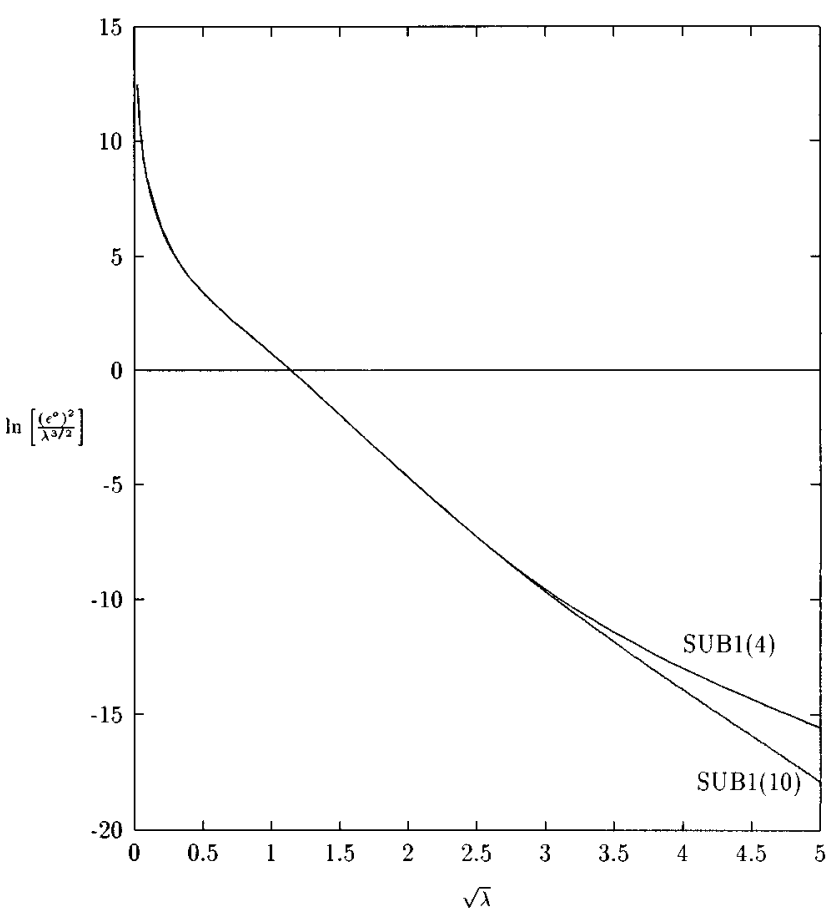

FIG. 2. Plot of $\ln \left[\left(\epsilon^{o}\right)^{2} / \lambda^{3 / 2}\right]$ against $\sqrt{\lambda}$ to show the exponential decrease of the glueball mass with increasing $\lambda$. Results for CCM calculations under the SUB1(4) and SUB1(10) approximations are shown.

reference state, and its use in conjunction with the "meanfield" reference state holds great promise.

The success of the CCM in its applications to the simple $\mathrm{U}(1)$ model in $(2+1) \mathrm{D}$ is very encouraging, and it will be very interesting to apply the formalism to the nontrivial case of $U(1)$ theory in $(3+1) D$, where a phase transition is expected, and also to non-Abelian models. In addition, the extension of our CCM analysis from the case of pure gauge theories to those including matter fields is also envisaged.

\section{ACKNOWLEDGMENTS}

We thank Y. Xian for useful discussions. One of us (R.F.B.) acknowledges the support of a research grant and another of us (S.J.B.) acknowledges financial support from the Engineering and Physical Sciences Research Council (EPSRC) of Great Britain.
[1] J. Kogut and L. Susskind, Phys. Rev. D 11, 395 (1975).

[2] J. W. Clark and E. Feenberg, Phys. Rev. 113, 388 (1959); H. W. Jackson and E. Feenberg, Ann. Phys. (N.Y.) 15, 266 (1961).

[3] F. Coester, Nucl. Phys. 7, 421 (1958); F. Coester and H. Kümmel, ibid. 17, 477 (1960).

[4] A. Dabringhaus, M. L. Ristig, and J. W. Clark, Phys. Rev. D 43, 1978 (1991).

[5] R. F. Bishop, A. S. Kendall, L. Y. Wong, and Y. Xian, Phys. Rev. D 48, 887 (1993).

[6] R. F. Bishop and Y. Xian, Acta Phys. Pol. B 3, 541 (1993).
[7] C. H. Llewellyn Smith and N. J. Watson, Phys. Lett. B 302, 463 (1993).

[8] C. J. Hamer and A. C. Irving, Z. Phys. C 27, 145 (1985).

[9] C. J. Hamer, J. Oitmaa, and Zheng Weihong, Phys. Rev. D 45, 4652 (1992).

[10] T. Höfsass and R. Horsley, Phys. Lett. 123B, 65 (1983).

[11] P. Suranyi, Phys. Lett. 122B, 279 (1983).

[12] P. Suranyi, Nucl. Phys. B225 [FS9], 77 (1983).

[13] D. W. Heys and D. R. Stump, Nucl. Phys. B257 [FS14], 19 (1985). 
[14] D. W. Heys and D. R. Stump, Nucl. Phys. B285 [FS19], 13 (1987).

[15] C. J. Morningstar, Phys. Rev. D 46, 824 (1993).

[16] R. F. Bishop, Theor. Chim. Acta 80, 95 (1991).

[17] T. Barnes and D. Kotchan, Phys. Rev. D 35, 1947 (1987).

[18] G. Blanch, in Handbook of Mathematical Functions, edited by M. Abramowitz and I. A. Stegun (U.S. GPO, Washington, D.C., 1964), p. 721.

[19] M. Creutz, Quarks, Gluons and Lattices (Cambridge University Press, Cambridge, England, 1983).

[20] A. C. Irving and C. J. Hamer, Nucl. Phys. B235 [FS11], 358 (1984)

[21] G. Lana, Phys. Rev. D 38, 1954 (1988).
[22] S. A. Chin, J. W. Negele, and S. E. Koonin, Ann. Phys. (N.Y.) 157, 140 (1984).

[23] I. Montvay and G. Münster, Quantum Fields on a Lattice (Cambridge University Press, Cambridge, England, 1994).

[24] D. Kotchan, Ph.D. thesis, University of Toronto, 1990, cited in Ref. [15].

[25] C. J. Hamer, K. C. Wang, and P. F. Price, Phys. Rev. D 50, 4693 (1994).

[26] N. J. Davidson and R. F. Bishop, in Lattice '94, Proceedings of the International Symposium, Bielefeld, Germany, 1994, edited by F. Karsch [Nucl. Phys. B (Proc. Suppl.) 42, 817 (1995)]. 